\title{
Touching Transport - A Case Study on Visualizing Metropolitan Public Transit on Interactive Tabletops
}

\author{
Till Nagel ${ }^{1,2}$, Martina Maitan ${ }^{3}$, Erik Duval ${ }^{2}$, Andrew Vande Moere ${ }^{4}$, \\ Joris KlerkX ${ }^{2}$, Kristian Kloeckl ${ }^{1}$, Carlo Ratti ${ }^{1}$ \\ ${ }^{1}$ MIT Senseable City Lab, \\ Massachusetts Institute of Technology \\ ${ }^{2}$ Department of Computer Science, \\ KU Leuven \\ ${ }^{3}$ Future Urban Mobility Group, \\ SMART \\ ${ }^{4}$ Department of Architecture \\ Urbanism and Planning, KU Leuven
}

\begin{abstract}
Due to recent technical developments, urban systems generate large and complex data sets. While visualizations have been used to make these accessible, often they are tailored to one specific group of users, typically the public or expert users. We present Touching Transport, an application that allows a diverse group of users to visually explore public transit data on a multi-touch tabletop. It provides multiple perspectives of the data and consists of three visualization modes conveying tempo-spatial patterns as map, time-series, and arc view. We exhibited our system publicly, and evaluated it in a lab study with three distinct user groups: citizens with knowledge of the local environment, experts in the domain of public transport, and non-experts with neither local nor domain knowledge. Our observations and evaluation results show we achieved our goals of both attracting visitors to explore the data while enabling gathering insights for both citizens and experts. We discuss the design considerations in developing our system, and describe our lessons learned in designing engaging tabletop visualizations.
\end{abstract}

\section{Categories and Subject Descriptors}

H.5.m [Information Interfaces and Presentation]: Misc.

\section{General Terms}

Design, Human Factors

\section{Keywords}

data visualization; multi-touch; exhibition; urban mobility

\section{INTRODUCTION}

In recent years, more and more urban data is digitally collected from municipal systems, sensors, and services. Various commercial initiatives in the field of smart cities from large technology companies such as IBM or Siemens work on

Permission to make digital or hard copies of all or part of this work for personal or classroom use is granted without fee provided that copies are not made or distributed for profit or commercial advantage and that copies bear this notice and the full citation on the first page. Copyrights for components of this work owned by others than ACM must be honored. Abstracting with credit is permitted. To copy otherwise, or republish, to post on servers or to redistribute to lists, requires prior specific permission and/or a fee. Request permissions from Permissions@acm.org.

AVI '14, May 27 - 30, 2014, Como, Italy

Copyright 2014 ACM 978-1-4503-2775-6/14/05...\$15.00.

http://dx.doi.org/10.1145/2598153.2598180 new possibilities of analyzing the urban environment for experts in public and private institutions. However, the question has been raised how to better involve the general public. Hill makes a case for active engaged citizens in order to unlock the potential of technology in a city [10]. While the increasing dissemination of mobile devices and location based services enable citizens employing urban data for tasks such as getting directions or finding a restaurant, these tools often lack supporting free-form exploration that can empower people to gain more general insights. In the larger process of working towards an informed discourse on smart cities, we deem reaching out to both experts and citizens with a tool that facilitates understanding of a city as an important step. One way of achieving that is to publicly exhibit visualizations of urban data in an urban demo. These urban demos [18] aim to provide visual and interactive access to information concealed in large urban data sets, engage public and industry partners in such operations, and collect feedback and ideas from users.

In this paper, we introduce Touching Transport, a case study enabling casual exploration of urban mobility in Singapore through a set of visualizations on a multi-touch tabletop. Our aim is to enable users to discover personally relevant stories and explore suitable subsets of data, while making visualizations more attractive and easy to use through playful interactions and a visually engaging design. Touching Transport is part of the LIVE Singapore! project [18], whose objective is to develop technologies and interface modalities to collect, combine and distribute large urban real time and historic data. In this case study, we focus on public transit data as it has various tempo-spatial characteristics typical in urban data, and is of interest to a wider audience.

After exhibiting our system at a symposium, we followed the semi-public deployment with a lab study informed by the exhibition to evaluate how and what kind of insights users get, and how they differ between different user groups.

In the following sections we will describe our design goals, introduce the system and explain our design decisions, report on public deployment and user study, and discuss our findings and lessons learned.

\section{DESIGN GOALS}

Experts from Singapore's land transport authority were continuously involved in the development through expert interviews, design goal discussions, and feedback rounds for visualization experiments. Furthermore, the design require- 
ments were informed by case studies on tabletop visualization for exhibitions [11, 12, 15, 27], and our own previous research $[21,22]$. We will describe the goals, explain how we aim to achieve them, and provide an overview on related work for each. The first two are general design goals of the urban demo, which influenced the latter three concerned with specific requirements for our system. As Touching Transport is intended for casual exploration at an exhibition, our emphasis is on attractive visual design, and simple interactivity, while still allowing different views into the data set.

\section{Enable access to data for different user groups.}

We see it as beneficial to bring together various stakeholders, and to bridge the gap between experts and laypeople for engagement regardless of their background. Interactive visualizations are an established way to communicate complex data to a larger audience [3]. They can provide a common language for different user groups. Visually, it should be appealing and easily understood without oversimplifying or distorting the information. Data needs to be personally meaningful to users for them to be explored in a casual context [26]. An interactive visualization of public transit should enable citizens to discover personally relevant patterns, for instance by looking at the time they are commuting, or by highlighting the areas around their neighborhood. Lastly, the system should employ interaction methods to support different exploration styles [12] in order to be usable by both laypeople and professionals with varying expertise.

\section{Support gathering insights.}

Informing citizens allows them to discuss public transit, and more generally participate in the debate of urban mobility. A side goal in our urban demo is to encourage users to express ideas and wishes. Our visualization system thus should support users in identifying interesting aspects, formulating questions, and ultimately gaining insights. In their recent position paper, Isenberg et al. [16] argue that data visualization on interactive surfaces can help making insight formation more attainable. Designing our system for a large tabletop aims to make data more accessible due to the ability to show detailed visualizations and to provide simple interactions.

\section{Entice curiosity.}

We aim to engage a broader audience and invite them to explore the system by enticing curiosity with attractive visualizations and large-scale tabletops. Interactive tabletops with large displays are seen as appealing to novice users [1], attract people's attention in public spaces [15], and support more casual visualization settings [16].

Visual and interface design are guided by principles of information aesthetics [19], with the goal to provide accurate data representation with easy-to-use interactivity. Aesthetics not only concern the visual form, but also aspects such as originality, innovation, and other subjective factors comprising the user experience [29]. While the novelty of a technique or system is not a value per se, it has been shown that novelty in design is an important factor to elicit aesthetic response from users [14]. Our goal was to use an attractive visual language resulting in a system easily understood and enjoyed by the users.

\section{Provide casual exploration.}

Our system is intended as a walk-up-and-use system so users can casually explore the data in an exhibition scenario, and aims to support contemplative usage [23] in a semi-public setting. The system should offer simple touch interactions so users can explore the data without learning complex gestures [16]. This lowers the cost for initial use [26]. Thus, we selected basic interaction methods based on well-established techniques fitting for tempo-spatial data. To invite users exploring the data for longer, the system should provide fluid interactions including high responsiveness and smooth view transitions [5]. Overall, we aim to provide an enjoyable user experience in order to engage visitors. And when they are accustomed and more experienced with the visualizations they might investigate urban mobility in more depth.

\section{Offer multiple perspectives.}

Multivariate data in the domain of urban mobility has many interdependent properties. Our goal is to enable users exploring the various facets, and discovering spatial and temporal patterns of public transit [6]. Thus, our system is intended to provide different visualizations, each offering a specific perspective into the data set. The challenge is to offer multiple linked views without being perceived as too complex in order to not discourage users from exploration [31].

\section{TOUCHING TRANSPORT}

Following our design goals we implemented Touching Transport as a functional prototype for a tabletop with multitouch capabilities ${ }^{1}$. It was built with Processing and the Unfolding Maps library. We developed it in an iterative design process, including various visualization experiments, discussions with experts and test users, and incorporating results therefrom into the system. In the following, we will describe the data set, the visualizations and interactions of the prototype, and discuss our design considerations.

\subsection{Public transit data}

In Singapore, passengers pay the distance-based fare by tapping in and out on boarding and alighting subways and buses. These actions are continuously recorded by smart card readers, and collected by the Land Transport Authority (LTA), the government agency responsible for road traffic and public transport. The data set consists of (i) geospatial (stations, routes, etc.), (ii) temporal (schedule, headways, etc.), and (iii) anonymized passenger data (tap ins and tap outs). From this, we derived further information such as origin-destination paths for each ride.

We used a subset from the various sources available within the Singapore public transport system, and settled on bus data for the prototype. With over 2 millions passengers per day, Singapore's bus network is an integral part of the system. We selected a sample of eight routes with different characteristics (e.g. express lines with few stops) to reflect a broad range of usage. These criteria were based on discussions with experts from the transport authority, and allowed us to examine the applicability for a set of transit data with different properties.

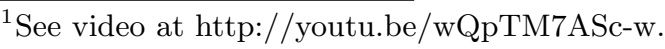



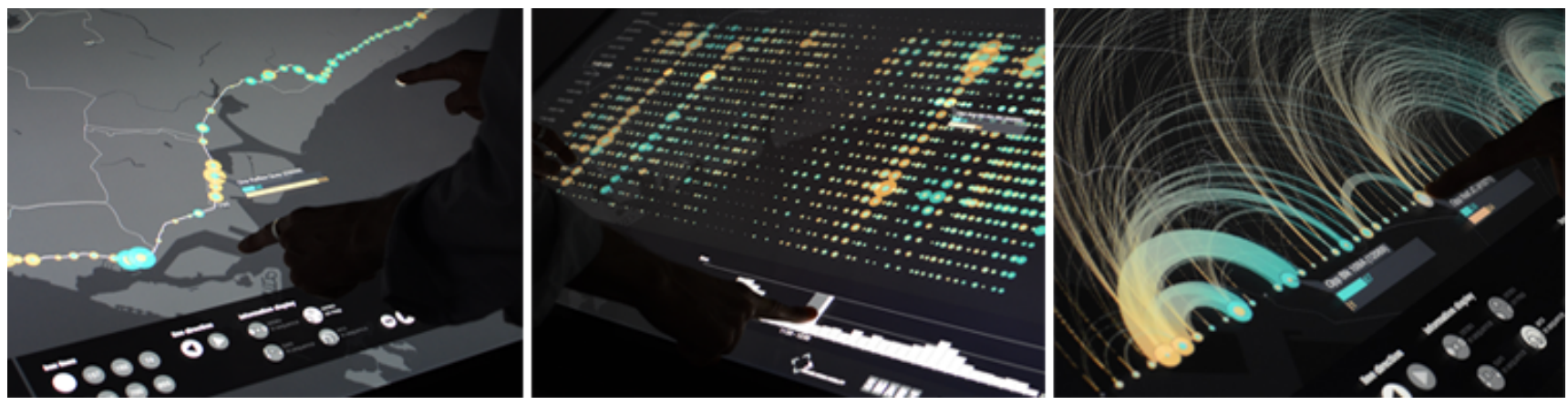

Figure 1: Multi-touch tabletop with (a) map, (b) time-series, and (c) arc visualization of passenger data.

\subsection{Three interactive visualizations}

Our system consist of three interlinked visualization modes, each supporting to look into different aspects of the data. It enables users to actively explore Singapore's bus network, and see where passengers get on and off, how people travel between the island's areas, and the way these patterns change throughout the day. While an interface at the bottom of the screen provides data filtering mechanisms, the most prominent part of the screen contains one of the three visualizations. Together with a large full-HD screen display, this design of showing a single visualization aims to attract casual users through its simplicity.

\subsubsection{Map View}

The map view shows passenger data for a selected bus line on a background map of Singapore (Figure 1a). For each stop boarding and alighting passengers are visualized as glyph consisting of two circles. The map view enables the understanding of passenger behavior in geo-spatial context, from analyzing and comparing single stops, to clusters of stops with similar characteristics, to neighborhoods and larger urban areas.

\subsubsection{Time-Series View}

The time-series view displays multiple charts of the same bus line for different times (Figure 1b). Each row shows all bus stops for a specific time of day. In a row, the stops of a bus line are displayed sequentially, with boarding and alighting passengers shown for each stop. The resulting columns visualize passengers for a single bus stop over time. Thus, users can observe different times at a glance, and identify tempo-spatial patterns, e.g. locate geo-spatial clusters of stops with similar properties over time.

A feature of this visualization is its adaptability to varying time ranges. Users can adapt the time range to see finer or coarser grained data aggregations. The number of rows (and the time span used for aggregating the displayed data) depends on the selected time range. Thus, users can identify hour-based temporal patterns, e.g. at what time a bus line begins getting crowded, as well as compare longer term ranges such as morning vs afternoon rush hours.

\subsubsection{Arcs View}

The arc view visualizes rides of a bus line (Figure 1c). Each arc signifies passengers traveling from one station to another, with the arc thickness indicating the number of passengers. This visualization enables users to see passenger flow, as well as connectivity of bus stops based on actual bus rides. The number of passengers are double encoded as arc transparency, so that the most frequented bus rides are easily discernible. Now, the characteristics of bus stops become visible, e.g. a stop with many thin arc connections, or a public transit hub with some strong connections.

\subsection{Visualization Design Considerations}

For displaying boarding and alighting passengers on the map, we use concentric circles both being scaled according to their value. We used a diverging binary color schema with turquoise for boarding and orange for alighting. In order to ease learning for casual users, we used the same encoding in the time-series view. While various other time-series graph techniques exist this ensured consistency.

While both map and arc modes support animating through time, the time-series view shows how passenger behavior evolve over time in a single view. We decided on the small multiples technique [28] which has been shown to be more effective than animation [24], and more efficient for comparisons across time series than combinations in one chart [17]. In order to reduce visual clutter and to support focusing on the sequential nature of a bus line, a linear layout for the bus stop sequences has been applied in this view. We opted against an equidistant positioning, where the screen distance between each two stops would have been equal, and chose a distance-based layout algorithm, where the screen distance between two stops is relative to their geographical distance. In that way, users still can recognize spatial groups of stops (e.g. city center), or low density line segments (e.g. express routes).

The arc visualization uses the same linear layout with the same glyphs per stop below, with the arcs showing directed rides above. While the results of a user study on visualizing directed edges [13] suggest using tapered connections, we could not apply a visual mapping with varying thickness as this was used for encoding the number of passengers. Thus, we mapped the direction to color gradient, with the same color schema as in all other visualizations, which also keeps consistency in regard to color coding.

\subsection{Interactions}

Touching Transport supports direct view manipulation as well as auxiliary controls. Users can directly interact within the visualizations, as well as filter the displayed data in the bottom bar, such as to select line direction, or change the time frame and duration. These interactions were based on well-established touch gestures, design patterns for data visualizations, and studies for map interactions [7]. 

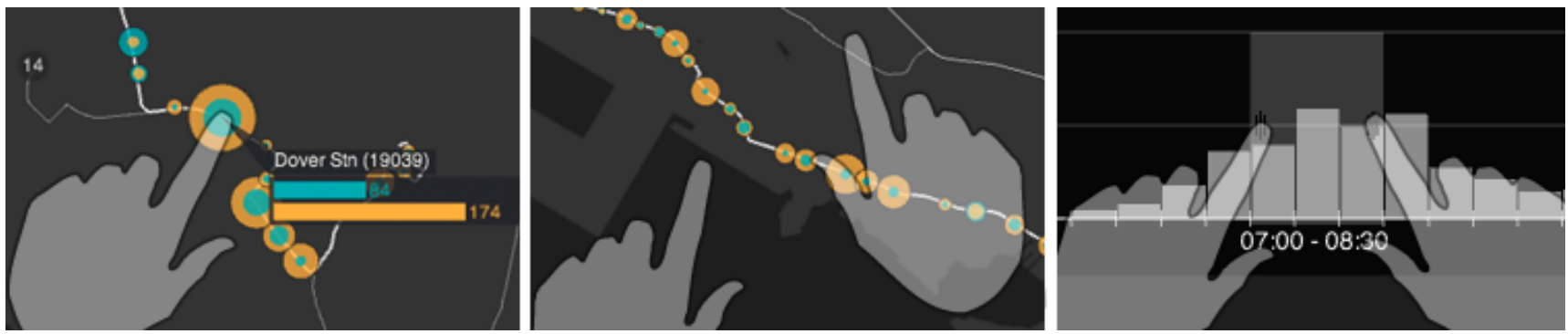

Figure 2: (a) Tap to select a bus stop, (b) free-form map manipulation, and (c) dual finger time range adaptation.

\subsubsection{Direct Interactions to select areas and stops}

In all three visualizations, users can tap on one (or multiple) bus stops to get details-on-demand with information on the stop and bar charts plus precise values for detailed comparison of the passenger data (Figure 2a). In the map view, users are able to select a region by panning and zooming the map with basic finger gestures. Users can drag to pan, rotate to re-orient, and pinch to zoom the map (Figure $2 \mathrm{~b}$ ). Even though more complex map manipulations are possible, we opted for simple navigation patterns in order to keep interaction complexity low.

\subsubsection{Auxiliary controls to filter data and time}

The bottom bar is visible at all times. A legend shows color coding, and visually explains how the data is mapped to its visual representation (Figure $3 \mathrm{~b}$ ). Tapping one of the bus route buttons (Figure 3c) selects the respective line in the main visualization. All data is shown with the currently selected filters applied.

The timeline contains an integrated histogram for passenger load and fluctuation (Figure 3e), and provides three functions: animation control, time selection, and time range selection. Tapping on the play button starts an animation through the day, giving users a quick impression of temporal travel patterns. Dragging the slider allows selecting any time of the day, for instance to compare morning and evening rush hour. Furthermore, users can investigate different time clusters, altering the range dynamically in 30 minute intervals by adjusting the time range slider (Figure 2c). This results in visual aggregations of the equivalent time interval. All time filtering methods update the displayed data in

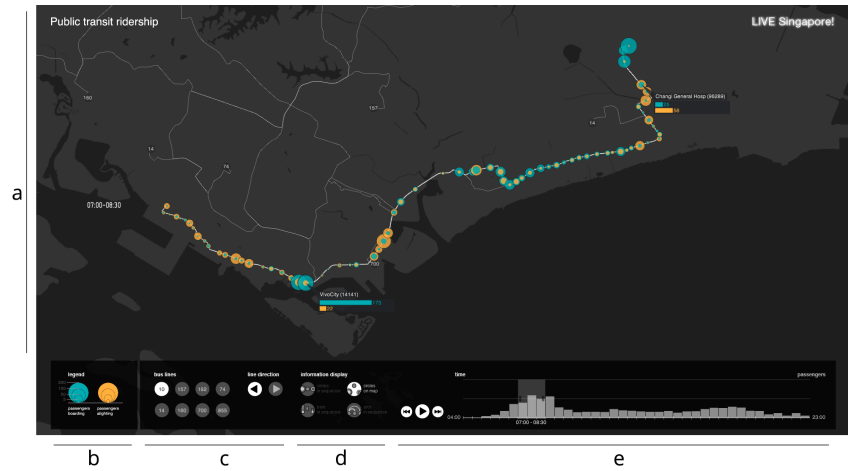

Figure 3: The interface with a) the main visualization (here: map), and the bottom bar containing b) legend, c) bus filters, d) visualization selector, and e) time range slider. all visualizations. All passenger data glyphs are updated instantaneously, and always reflect the currently selected time range. Their visual properties switch to the next state in an animated transition enabling users to visually track the change, and to easier identify changes in the behavior of passengers.

\subsection{Switching visualization modes}

Users can switch between the three visualization modes (Figure 3d). Showing one visualization at a time provides a simple entry point to exploring the data. We did not opt for the more classic approach of having multiple coordinated views [4] in order to reduce visual complexity, and not overload a casual user with too much information. In their study on serendipitous discoveries through information visualization, Thudt et al. [27] used multiple views, describe how some of their users felt affected by its visual complexity, and suggest showing only one visualization at a time as a potential remedy. Hence, in Touching Transport only one visualization is displayed in the main screen area. Yet, all three visualizations are interlinked. Selected bus stops in the main view, as well as all data filters chosen in the bottom panel stay consistent in all modes to maintain context, and to enable comparing the same data in different views [31].

When a user switches to another visualization the application animates between the old and the new view. These transitions support the perception of changes between different data graphics [8]. When, for instance, a user switches from arcs to map view, the arcs fade out and the background map fades in, while the visual representations of all bus stops animate to their new position, resulting in the whole route morphing from linear to geographical order. In this way, our system supports users understanding patterns in different views while keeping the spatial context.

To summarize, the goals of the three visualizations are (i) to show spatial patterns, and provide an interface to select areas of interest, (ii) to show tempo-spatial clusters, and allow comparing different times at a glance, and (iii) to show flows of passengers, and connectivity between bus stops and urban areas. Switching between these lets users focus on one view, and still enables them to select the best fitting one in order to gain insights based on different data properties.

\section{DEPLOYMENT AT EXHIBITION}

Touching Transport has been exhibited at an urban mobility symposium at the National University Singapore (see Figure 4). Open to researchers, civic employees, and invited citizens, this allowed us to informally observe usage in a non-study setting. 


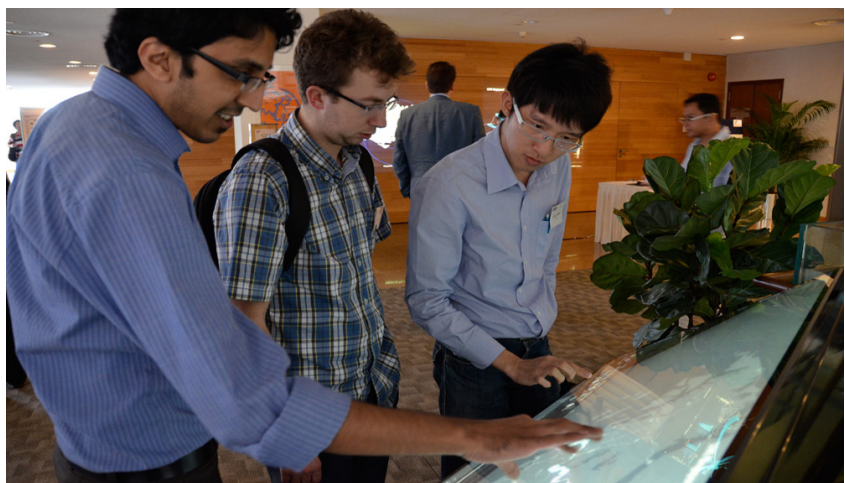

Figure 4: Visitors exploring Touching Transport at an exhibition in Singapore.

In two days over hundred visitors interacted with our system. The multi-touch table had been set up with a slight inclination in order to attract passers-by. Many visitors noticed the large screen even from a distance, and approached to look at the visualizations. Some people first watched it for a while, some directly started tapping the surface and exploring the system. We noticed how some users tried simply touching the screen without any apparent target, assumedly to see if the system is interactive. These were more inclined to explore it further when the map was shown, as this view reacts wherever the user touched the screen.

Visitors had have few problems interacting with the visualizations, were able to switch bus lines and view modes, and dragging the time slider. Although our system is intended for single user interaction, the large interactive screen attracted visitors when others already were gathering around the table. We have observed this honey pot effect [2] independent from whether the earlier visitors were only watching and/or discussing, or already actively exploring the system.

Visitors seemed to like the visualizations based on their facial expressions or their feedback in informal discussions. We also observed persons coming back and bringing friends and colleagues along to show them specific stories they had found earlier. Urban demos act as a catalyst bringing together actors from different fields, and enabling them having an informed debate. We have seen various discussions taking place around the tabletop, often within groups of strangers.

Exhibiting Touching Transport at the symposium was a success from our and our partners view: (i) Members from the LTA have expressed their wish to deploy the prototype in the foyer at their main office, as they highly liked how visitors were attracted to the tabletop. (ii) The LTA has asked us since to help bring an extended version of the system in-house. We did not expect this as our system was intended for casual information visualization, and the authority possess and utilize their own specialized analytic tools. They explained that our visualizations were more engaging to use, and that multi-touch interactions could lower the access barrier for non-IT experts. (iii) Currently, Touching Transport is exhibited in our lab in Singapore, and demonstrated to visitors to act as starting point for discussing the value of data visualizations in developing smart cities.

\section{EVALUATION STUDY}

Information visualization systems for visual analysis commonly aim to provide expert level insights. Visitors in exhi- bitions may not have clearly defined questions in mind, but explore visualizations based on spontaneous interest [15]. We observed visitors investigating some questions and hunches while playing with the system in the exhibition. In informal discussions we learned they occasionally arrived at insights about specific parts of urban mobility. We found it difficult to record users gathering insights in an exhibition setting as we observed visitors exploring Touching Transport contemplatively. Even when discussing their findings with others, users not necessarily explained their inner monologue. Thus, after the first public exhibition, we designed a user study to investigate in detail how participants use the system while following ideas and questions. We evaluated how Touching Transport supports different user groups, collect what kind of insights users could gain, and verify that different levels of understanding of urban mobility can be reached. We report on the setup, the participant groups, and insights and satisfaction results in this section.

\subsection{Study design}

The study consisted of five parts: (1) The introduction with explanation of the study and the system, (2) a period to freely explore the application to get accustomed with it, (3) the main part where the participant had to come up with insights, (4) a questionnaire to measure satisfaction, and (5) an open discussion for further feedback and suggestions. For parts (2) and (3) we asked the participant to think-aloud, and recorded the audio, while the interviewer observed and recorded the behavior of the participants, and how they interacted with the system. Then, we asked for three insights in three separated sub-sessions of 5 minutes each (and not for just insights in one large session). We intended the three sub-sessions to encourage participants to come up with different findings and/or to use different visualizations.

Each session had one participant. The study was performed in a lab setting, i.e. a room with the tabletop with adjusted light settings and no disruptions. This was done due to allow participants to discuss occurring usability problems, and to motivate reporting more expansive insights via the think-aloud method. Overall, including the (4) post-test satisfaction survey and the (5) open discussion, a session took between 40-60 minutes.

\subsection{Participants}

We tested Touching Transport with 27 participants from three user groups: citizens with knowledge of the local environment (LOC), experts in the domain of urban mobility (EXP), and non-experts with neither local nor domain knowledge (NON). For the first group (LOC), we recruited eleven members from our institution in Singapore (five members of non-research staff and six researchers from other groups). For the second group (EXP), we had six participants from the transport authority. For the third group (NON) we recruited ten students from KU Leuven, a university in a medium-sized European city, with majors in computer science or architecture. Overall, we had 6 female and 21 male participants, aged 18 to 40 years (median: 28).

All participants had prior experience with touch devices, and eight $(29 \%)$ with large-scale multi-touch devices. They had to self-assess their experience with data visualization, and their knowledge about Singapore and public transit. Expert participants had most experience with visualization, and highest knowledge of public transit in comparison to 

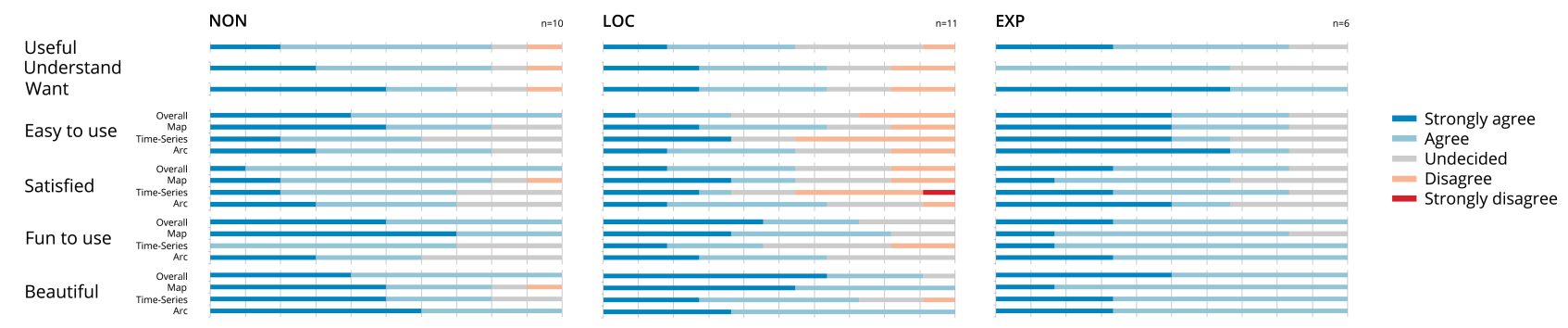

Figure 5: Questionnaire results for each user group as normalized stacked bar chart, grouped by agreement (blue) and disagreement (red). The lower charts each show four bars for overall system, map, time-series, and arc visualization.

the other two groups. Experts also estimated their knowledge about Singapore highest, with local citizens second, and Leuven students last. The results are according to our expectations and verify the classification of our user groups.

\subsection{Insights}

To verify whether users were able to gain more advanced levels of knowledge, we followed the suggestion of Vande Moere et al. to also request reports of meaning instead of only asking for facts [30]. We codified and classified insights participants made (see Table 1) while exploring the data into one of the following three categories, and for each describe exemplary insights participants made.

\subsubsection{Basic insights}

The first category consists of insights into elementary spatial and visual properties. Participants understood the shape of Singapore and its topological features. They could employ the map to relate visual representations of bus stops to their positions, and understand spatial relations such as estimations of their distance. All participants were able to identify starting and ending stops of a line. Furthermore, they recognized outstanding stops of a bus line, and for instance found the ones with large amounts of passenger flow. They were also able to compare two elements in a single visualization, and for instance identify the lesser used bus stop out of two.

\subsubsection{Medium insights}

The second category consists of insights into more advanced spatial patterns, and basic tempo-spatial patterns. Participants were able to recognize spatial clusters of similar bus stops. Furthermore, participants compared different elements over time, and were able to see basic commuting patterns. Most participants could correctly deduce dwelling and working areas, based on the amount of passengers boarding in one and alighting in the other in the morning hours. Even participants from NON with no local knowledge could identify areas in this way. Participants found the morning peak to be shorter in time, while the evening peak to be spread out by using the time slider, and analyzing the temporal patterns in the passenger histogram. Some participants went on and inferred that this might be due to a wider duration of time in which people leave the office, or are active at night

Table 1: Insights in each category per participant group

\begin{tabular}{l|ccccc} 
& Basic & Medium & Advanced & Total & Average \\
\hline NON (10) & 16 & 8 & 4 & 28 & 2.8 \\
LOC (11) & 12 & 17 & 7 & 36 & 3.3 \\
EXP (6) & 4 & 5 & 12 & 21 & 3.5
\end{tabular}

such as running errands or having dinner before retiring for the day.

\subsubsection{Advanced insights}

The third category consists of insights into more complex tempo-spatial patterns. Here, participants needed to filter the data, for instance by updating the time-range or selecting different bus lines. In this way, they could identify which of multiple bus lines had most passengers at a specific time. Some also used this to further investigate commuting patterns, and discovered how these are inverted in the evening. Participants from Singapore (LOC and EXP) used their local knowledge to identify transfer stations, such as bus stops which are nearby metro stations. Five out of six expert and half of the citizen participants were able to associate movements with areas of Singapore. Some created hypotheses on more specific reasons for passenger flow, for instance that afternoon people travel towards VivoCity, a shopping mall in Singapore.

Furthermore, advanced insights were gathered by comparing the three different visualizations. Participants switched between views to investigate if there were relationships between spatial areas. They were for instance able to see that a specific stop (whose large glyph on the map indicated many alighting passengers) was getting passengers from various stops in the arc view, and deduced that these were passengers riding from their homes towards a transport hub. Participants from EXP were able to apply their expertise and linked the visualized data with specific prior knowledge in order to gather more advanced insights.

\subsubsection{Summary}

These results suggest our system empowers audiences with divergent backgrounds acquiring a better understanding of urban mobility. Overall, our prototype supports users understanding public transit by getting various insights. In our study, non-expert participants were able to gather a general understanding, citizens a deeper sense of their city, and experts higher levels of insights.

\subsection{Satisfaction}

At the end of the study participants completed a brief post-test satisfaction survey with 21 questions. The survey was based on Lund's USE Questionnaire [20], and used a 5-point Likert scale ranging from Strongly agree (5) to Strongly disagree (1).

Most participants found Touching Transport useful, and agreed or strongly agreed it helps them understand public transit in general (8/10 NON, 6/11 LOC, 4/6 EXP). They were satisfied with the system and its ease-of-use, found it 
fun to use (24 out of 27 ), and aesthetically pleasing (26 out of 27) (see Figure 5 for details). While the results for all three groups were mostly similar, some areas had differences between groups. Firstly, citizens (LOC) were less satisfied with the ease-of-use of the overall system and with each visualization compared to the other groups (3.18 vs NON:4.4, EXP:4.3). One explanation is that our citizen participants had lower prior experience with data visualization. Secondly, all groups agreed to the statement the overall system was fun to use, and beautiful. However, the fun-to-use results varied in regard to the different views. Non-experts (NON) preferred the map the most (4.7 vs EXP: 4.0, LOC: 4.1), while experts favoured the time-series (EXP: 4.2 vs NON: 3.7, LOC: 3.4). As both groups rated the ease-of-use for the map visualization equally, this difference could indicate that non-experts prefer familiarity as they are most accustomed to reading and interacting with maps. On the other hand, experts in the field of public transit seem to prefer abstract visualization methods as it allows them to view more complex aspects of the data.

\section{DISCUSSION}

In the following we discuss and generalize some of the lessons we learned in designing our system in order to inform future case studies with the goal of enabling insights into complex data sets.

\section{Crafting aesthetics: Visual style and Responsiveness}

In the field of information visualization there typically is a distincation between systems with a high appeal to communicate stories to a general audience on the one hand, and expert systems to support analyzing data and generating insights on the other. While visualizations in public exhibitions have been studied for casual users, our goal, in contrast, was to enable both experts and citizens gaining insights. The focus of our research was to employ established visualizations adapted to a specific set of urban data. Our contribution lies in their aesthetic and usable composition, and the description of our design considerations.

We tried balancing appeal and accuracy in our visualizations. A visual design with the sole goal of being aesthetic may have a negative impact on readability of precise data values. While multiple expert participants autonomously verified the general validity of our visualizations with their prior knowledge, the intent of casual visualizations is not to come to crystalized conclusion [23], nor exact qualitiative estimation of values for each visual element [15]. Based on people's insights we conclude that our system enables people to understand different patterns correctly. We have shown that it is possible to design an exploration tool that helps a diverse range of users gaining new insights, while attracting casual users with an appealing style.

In both the exhibition as well as the lab study, users were pleased with the fluidity of the interactions, and liked the highly responsive interactivity. Some users expressed their joy about how "snappy" the time slider felt. Touching Transport supports traversing the visualization pipeline with such performance that every filtering of the data or adaptation of the view is instantly reflected $(<0.3 \mathrm{~s})$ in the graphical display. While it might be difficult to provide immediate response for voluminous and complex datasets [25], investing time and effort in the technical implementation of the system contributes to a enjoyable user experience. Our study results show that carefully designing for performance fosters keeping people engaged with a visualization system in a casual setting.

In summary, Touching Transport has been found to be enjoyable and aesthetically pleasing, and to foster an engaging user experience. Thus, we underline the importance of craft in designing high quality visualization systems.

\section{Multiple coordinated vs single modal views}

Touching Transport shows one visualization at a time, rather than all three simultaneously, in order to lower visual complexity for casual users. We observed users switching views to verify a hypothesis about the displayed data, for instance from arc to map view (and vice versa) in order to understand the spatial properties of the visualized bus route. While we did not evaluate through a comparative study whether the benefit of coordinated multiple views in visualizations outweighs the possible distractions they might create [9], with our case study we demonstrated that switching modes work, and can provide multiple perspectives to support obtaining insights. Moreover, the cognitive load of switching views can be lowered with animation. Participants in our study highly liked the transitions between the three visualization modes, and also found it to ease following currently focused data elements. We believe the trade-off of mentally maintaining context is worth the simplicity of a single view. Having one view at a time allowed us furthermore to attract users with one visualization they are familiar with. In our case we utilized the map view as simple entry point to invite visitors to start exploring.

\section{Exhibiting prototypes as part of the design process}

A further contribution are the results from both a public deployment and a controlled evaluation study of the system in use. In our design process of creating and evaluating an urban demo, our in-the-wild study primed the lab study. Our contribution lies in the reflection on why we chose this methodological setup, and in the systematic study with three groups of users. We described the differences and similarities in their behaviour, and the varying levels of insights they made.

Citizens and non-experts expressed their wishes for similar visualizations helping them to improve personal travel, while experts asked for having such system for visual analytics to support both planning and real-time control. The fact that users propose such features demonstrates that the system works for idea generation. Our urban demo helped people think about yet new ways of understanding public transit that go beyond the system's current possibilities.

\section{CONCLUSION}

With our case study on public transit data, we demonstrated how interactive visualizations on tabletops can contribute to the emerging field of smart cities. The three visualization modes facilitate getting different perspectives on the data set, while the fluid interactivity integrates them into a unified user experience. Together, they provide people visual and tangible access to information about the public transit network, and enable understanding some of the vital dynamics of their city. A deployment at an exhibition and the results from our user study suggest that our design goals were largely met: visitors were attracted to the system and casually explored the data. Participants of our study were 
able to obtain insights, ranging from understanding basics such as transport hubs to more advanced such as commuting patterns.

While supporting concrete citizen participation is an important subject, it was beyond the scope of our research. In this paper, we argue that a well-designed system visualizing urban data can inform citizens and enable them to gain insights. Our intention was to create an urban demo to publicly exhibit visualizations in order to encourage discussion and gather feedback on mobility. Yet, our application is but one step in the larger process of bringing together different stakeholders of a city, and ultimately encouraging participation.

We see the lessons learned to be generalizable for other tempo-spatial data sets aimed at a diverse audience. Within Live Singapore, we are currently developing a system allowing citizens to combine and explore different urban data sets.

\section{ACKNOWLEDGMENTS}

We would like to thank Oliver Senn for his excellent support in data preparation and analytics. We also thank the participants of our user study, and the Singapore Land Transport Authority for providing data and expert feedback.

\section{REFERENCES}

[1] H. Benko, M. R. Morris, A. B. Brush, and A. Wilson. Insights on Interactive Tabletops: A Survey of Researchers and Developers. Microsoft Research Technical Report MSR-TR-2009-22, 2009.

[2] H. Brignull and Y. Rogers. Enticing people to interact with large public displays in public spaces. In Proc. of INTERACT, volume 3, pages 17-24, 2003.

[3] A. Cairo. The Functional Art. New Riders, 2012.

[4] C. Collins and S. Carpendale. Vislink: Revealing relationships amongst visualizations. IEEE TVCG, 13(6):1192-1199, 2007.

[5] N. Elmqvist, A. Vande Moere, H.-C. Jetter, D. Cernea, H. Reiterer, and T. Jankun-Kelly. Fluid interaction for information visualization. Information Visualization, 10(4):327-340, 2011.

[6] D. Fairbairn. Geovisualization Issues in Public Transport Applications. In Exploring Geovisualization, pages 513-528. Elsevier, 2005.

[7] M. Harrower and B. Sheesley. Designing Better Map Interfaces: A Framework for Panning and Zooming. Transactions in GIS, 9(2):77-89, 2005.

[8] J. Heer and G. G. Robertson. Animated transitions in statistical data graphics. IEEE TVCG, 13(6):1240-1247, 2007.

[9] J. Heer, F. van Ham, S. Carpendale, C. Weaver, and P. Isenberg. Creation and collaboration: Engaging new audiences for information visualization. In Information Visualization, pages 92-133. Springer, 2008.

[10] D. Hill. The City that Smart Citizens Built. Archis, 4, 2012.

[11] U. Hinrichs and S. Carpendale. Gestures in the wild: studying multi-touch gesture sequences on interactive tabletop exhibits. In Proc. CHI 2011, pages 3023-3032. ACM, 2011.

[12] U. Hinrichs, H. Schmidt, and S. Carpendale. EMDialog: Bringing Information Visualization into the Museum. IEEE TVCG, 14(6):1181-1188, 2008.
[13] D. Holten and J. J. van Wijk. A User Study on Visualizing Directed Edges in Graphs. In Proc. CHI 2009, pages 2299-2308. ACM, 2009.

[14] W. Hung and L. Chen. Effects of novelty and its dimensions on aesthetic preference in product design. International Journal of Design, 6(2):81-90, 2012.

[15] P. Isenberg, U. Hinrichs, M. Hancock, and S. Carpendale. Digital tables for collaborative information exploration. In Tabletops-Horizontal Interactive Displays, pages 387-405. Springer, 2010.

[16] P. Isenberg, T. Isenberg, T. Hesselmann, B. Lee, U. Von Zadow, and A. Tang. Data Visualization on Interactive Surfaces: A Research Agenda. IEEE $C G \mathscr{E} A, 33(2), 2013$.

[17] W. Javed, B. McDonnel, and N. Elmqvist. Graphical perception of multiple time series. IEEE TVCG, 16(6):927-934, 2010.

[18] K. Kloeckl, O. Senn, and C. Ratti. Enabling the Real-Time City: LIVE Singapore! Journal of Urban Technology, 19(2):89-112, 2012.

[19] A. Lau and A. Vande Moere. Towards a model of information aesthetics in information visualization. In Proc. $I V^{\prime} 07$, pages 87-92. IEEE, 2007.

[20] A. M. Lund. Measuring usability with the use questionnaire. Usability interface, 8(2):3-6, 2001.

[21] T. Nagel, E. Duval, and A. Vande Moere. Interactive Exploration of Geospatial Network Visualization. In Ext. Abstracts CHI 2012, pages 557-572. ACM, 2012.

[22] T. Nagel, F. Heidmann, M. Condotta, and E. Duval. Venice Unfolding: A Tangible User Interface for Exploring Faceted Data in a Geographical Context. In Proc. NordiCHI 2010, pages 743-746. ACM, 2010.

[23] Z. Pousman, J. T. Stasko, and M. Mateas. Casual information visualization: Depictions of data in everyday life. IEEE TVCG, 13(6):1145-1152, 2007.

[24] G. Robertson, R. Fernandez, D. Fisher, B. Lee, and J. Stasko. Effectiveness of animation in trend visualization. IEEE TVCG, 14(6):1325-1332, 2008.

[25] R. E. Roth. Interactive maps: What we know and what we need to know. Journal of Spatial Information Science, 2013(6):59-115, 2013.

[26] D. Sprague and M. Tory. Exploring how and why people use visualizations in casual contexts: Modeling user goals and regulated motivations. Information Visualization, 11(2):106-123, 2012.

[27] A. Thudt, U. Hinrichs, and S. Carpendale. The Bohemian Bookshelf: Supporting Serendipitous Book Discoveries through Information Visualization. In Proc. CHI 2012, pages 1461-1470. ACM, 2012.

[28] E. R. Tufte. Envisioning Information. Graphics Press, 1990.

[29] A. Vande Moere and H. Purchase. On the role of design in information visualization. Information Visualization, 10(4):356-371, 2011.

[30] A. Vande Moere, M. Tomitsch, C. Wimmer, B. Christoph, and T. Grechenig. Evaluating the Effect of Style in Information Visualization. IEEE TVCG, 18(12):2739-2748, 2012.

[31] M. Q. Wang Baldonado, A. Woodruff, and A. Kuchinsky. Guidelines for Using Multiple Views in InfoVis. In Proc. AVI, pages 110-119. ACM, 2000. 